\title{
Persistence of MRI changes in central pontine myelinolysis
}
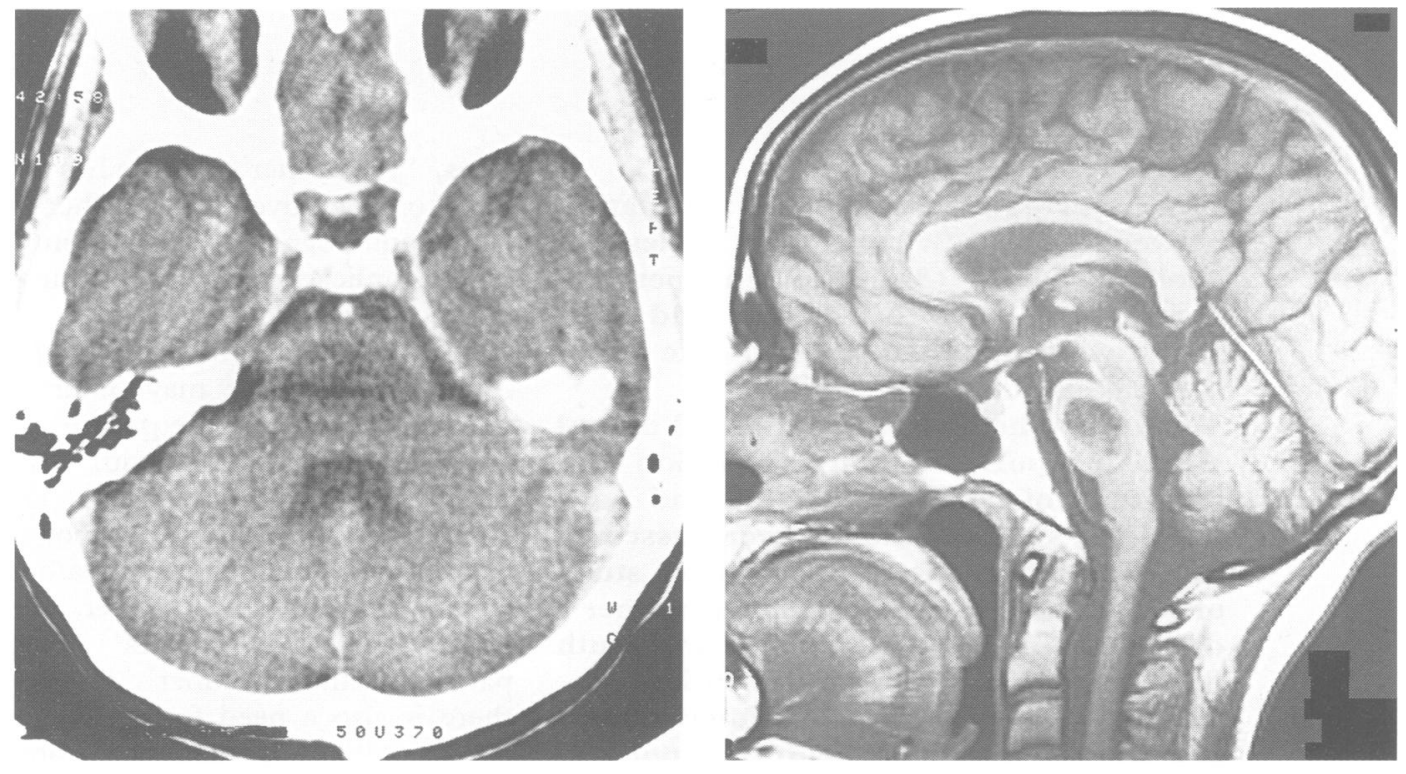

A

B
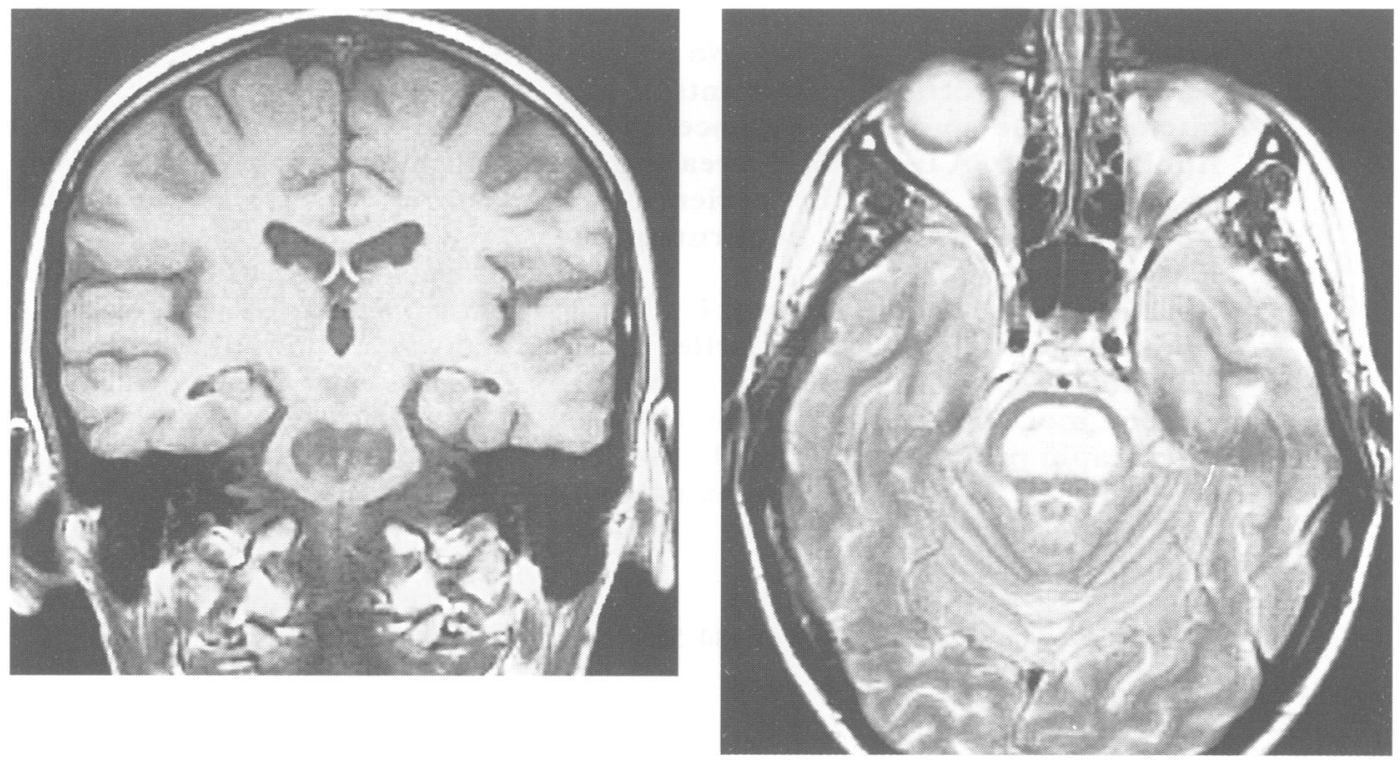

D

A 21 year old woman with a four year history of anorexia, bulimia, depression, and alcoholic liver disease presented elsewhere in stupor, incontinent of urine and with extensor plantar responses. Serum sodium concentration was 111 $\mathrm{mmol} / \mathrm{l}$ which rose to $122 \mathrm{mmol} / \mathrm{l}$ at 20 hours and $134 \mathrm{mmol} / 1$ at 92 hours after commencement of an intravenous infusion of $2 \mathrm{~N}$ saline. She improved but was readmitted nine days later with dysarthria, finger-nose ataxia, and poor tandem gait. Brain CT (figure, A) showed extensive low density in the central pons. Sagittal and coronal T1 (figure, B, C) and axial T2 weighted MRI (figure, D) after 10 weeks showed abnormal signal in the pons with an intact rim around the surface, as is seen in pathological specimens. ${ }^{1}$ Four months later she had made a full neurological recovery, but repeat MRI at seven months showed no change. Persisting pontine defects on $M^{2} I^{2}$ must be considered if these patients need subsequent imaging for possibly unrelated neurological illness.

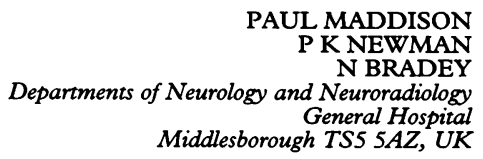

1 Allen IV, Kirk J. Demyelinating diseases. In: Hume AJ, Duchen LW, eds. Greenfield's neuropathology. 5th ed. Edward Arnold, 1992:497-8. 2 Thompson PD, Miller D, Gledhill RF, Rossor MN. Magnetic resonance imaging in central pontine myelinolysis. $\mathcal{f}$ Neurol Neurosurg Psychiatry 